AperTO - Archivio Istituzionale Open Access dell'Università di Torino

\title{
Geometry of particle paths in turbulent flows
}

\section{This is the author's manuscript}

Original Citation:

Availability:

This version is available http://hdl.handle.net/2318/1617922

since 2016-11-29T10:11:11Z

Published version:

DOI: $10.1080 / 14685240600860923$

Terms of use:

Open Access

Anyone can freely access the full text of works made available as "Open Access". Works made available under a Creative Commons license can be used according to the terms and conditions of said license. Use of all other works requires consent of the right holder (author or publisher) if not exempted from copyright protection by the applicable law. 


\title{
Geometry of particle paths in turbulent flows
}

\author{
W. BRAUN*, F. DE LILLO and B. ECKHARDT \\ Fachbereich Physik, Philipps-Universität Marburg, D-35032 Marburg, Germany
}

\begin{abstract}
We use the intrinsic geometrical characteristics of space curves, i.e. curvature and torsion, to describe the paths of particles passively advected in a turbulent flow. We find that curvature increases with the Reynolds number and that the maxima in vorticity and curvature times local velocity are linearly correlated. Fluctuations around the maximal values in runs at a fixed Reynolds number are only weakly correlated, and the distributions are fairly wide, so that it is not possible to conclude that a tightly wound spiral has to correspond to a region of high vorticity.
\end{abstract}

Keywords: Lagrangian statistics; Chaotic advection

\section{Introduction}

Optical and acoustical techniques now allow the tracking of isolated particles in turbulent flows with a time and space resolution that reaches all the way down to the Kolmogorov scales [1-5]. Numerical differentiation of these trajectories gives information about the Lagrangian velocity and acceleration of the particles [1-6], and the data have raised many interesting questions about the statistical properties of these quantities [10,11]. The tracking of several particles and their mutual relation is of interest for pair dispersion [1, 12] and understanding of anomalies in the structure functions $[13,14]$. Velocity information from several particles can also be used to obtain information about spatial variations and gradients [15].

The path of a particle also defines a curve in 3D space, and the intrinsic geometric characterization of these curves calls for the determination of curvature and torsion. The prototypical space curve is a spiral, and the association with swirling around a vortex already suggests that curvature and torsion contain information about vorticity, i.e. spatial gradients, and that perhaps regions with high curvature are in intense vorticity regions. In the following, we will explore this relation between the space curve characteristics curvature and torsion and flow properties such as vorticity and dissipation, in order to probe the correlation between spirals and vorticity and to relate the gradients in the curves to statistical small-scale quantities. Eventually, such relations may be relevant for the intermittent behaviour in turbulent flows [16].

After a summary of the basic features in section 2, we turn to a discussion of the flow properties in a stationary ABC flow (section 3), followed by the analysis of numerical data from turbulent DNS in section 4 . We conclude with a summary in section 5.

*Corresponding author. E-mail: Bruno.Eckhardt@Physik.Uni-Marburg.DE

Journal of Turbulence

ISSN: 1468-5248 (online only) (c) 2006 Taylor \& Francis

http://www.tandf.co.uk/journals

DOI: $10.1080 / 14685240600860923$ 


\section{Basics}

With $\mathbf{u}(\mathbf{x}, t)$ the velocity field at position $\mathbf{x}$ and time $t$, the equation of motion for a passively advected particle is $\dot{\mathbf{x}}_{P}=\mathbf{u}\left(\mathbf{x}_{P}, t\right)$. For the evaluation of geometrical properties, we need the total change in velocities,

$$
\begin{aligned}
\frac{\mathrm{d} \mathbf{u}}{\mathrm{d} t} & =\partial_{t} \mathbf{u}+\mathbf{u} \cdot \nabla \mathbf{u} \\
\frac{\mathrm{d}^{2} \mathbf{u}}{\mathrm{d} t^{2}} & =\partial_{t} \dot{\mathbf{u}}+\mathbf{u} \cdot \nabla \dot{\mathbf{u}} \\
& =\partial_{t}^{2} \mathbf{u}+\left(\partial_{t} \mathbf{u}\right) \cdot \nabla \mathbf{u}+2 \mathbf{u} \cdot \partial_{t} \nabla \mathbf{u}+\mathbf{u} \cdot \nabla(\mathbf{u} \cdot \nabla \mathbf{u}) .
\end{aligned}
$$

We can now introduce the set of accompanying tangent, normal and bi-normal vectors (see figure 1). The tangential vector is defined as the derivative with respect to the path length $s$ of the curve:

$$
\mathbf{t}=\frac{\mathrm{d} \mathbf{r}}{\mathrm{d} s}=\frac{\mathbf{u}}{|\mathbf{u}|}
$$

The normal vector points in the direction of the acceleration perpendicular to the tangential:

$$
\mathbf{n}=\frac{1}{\kappa} \frac{d^{2} \mathbf{r}}{d s^{2}}=\frac{1}{\kappa} \frac{\mathrm{d} \mathbf{t}}{\mathrm{d} s}
$$

with the curvature

$$
\kappa=\frac{\sqrt{\dot{\mathbf{u}} \cdot \dot{\mathbf{u}}-(\mathbf{t} \cdot \dot{\mathbf{u}})^{2}}}{\mathbf{u} \cdot \mathbf{u}},
$$

where here the dot denotes the total time derivative (1). The set is completed with the bi-normal vector, which is perpendicular to the tangential and normal vector:

$$
\mathbf{b}=\mathbf{t} \times \mathbf{n} .
$$

The change of all three vectors along a trajectory is described by Frenet's formulae:

$$
\begin{aligned}
& \frac{\mathrm{d} \mathbf{t}}{\mathrm{d} s}=\kappa \mathbf{n}, \\
& \frac{\mathrm{d} \mathbf{n}}{\mathrm{d} s}=\tau \mathbf{b}-\kappa \mathbf{t},
\end{aligned}
$$

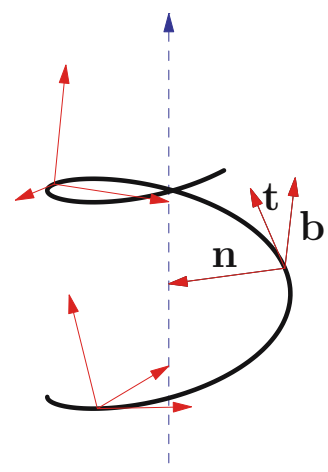

Figure 1. A space curve and the tangential vector $\mathbf{t}$, the normal vector $\mathbf{n}$ pointing towards the centre of a spiral and the bi-normal vector $\mathbf{b}$, perpendicular to both $\mathbf{t}$ and $\mathbf{n}$. 


$$
\frac{\mathrm{d} \mathbf{b}}{\mathrm{d} s}=-\tau \mathbf{n} .
$$

The scalar parameters are the curvature, either calculated from (6) or from

$$
\kappa=\frac{|\mathbf{u} \times \dot{\mathbf{u}}|}{|\mathbf{u}|^{3}}
$$

and the torsion $\tau$,

$$
\tau=\frac{\mathbf{u} \cdot(\dot{\mathbf{u}} \times \ddot{\mathbf{u}})}{(\mathbf{u} \cdot \mathbf{u})^{3} \kappa^{2}} .
$$

The curvature radius and torsion radius are related to curvature and torsion according to

$$
\rho=1 / \kappa, \quad \sigma=1 / \tau .
$$

To illustrate the meaning of these quantities, consider the spiral

$$
\mathbf{r}(t)=\left(\begin{array}{c}
R \cos \Omega t \\
R \sin \Omega t \\
\beta \Omega t
\end{array}\right), \quad \mathbf{u}(x, y)=\Omega\left(\begin{array}{c}
-y \\
x \\
\beta
\end{array}\right)
$$

as a normal form for a curve with constant curvature and torsion. Then

$$
\kappa=\frac{R}{R^{2}+\beta^{2}}, \quad \tau=\frac{\beta}{R^{2}+\beta^{2}}, \quad u=\Omega \sqrt{R^{2}+\beta^{2}},
$$

and the parameters of the spiral can be recovered as

$$
R=\frac{\kappa}{\kappa^{2}+\tau^{2}}, \quad \beta=\frac{\tau}{\kappa^{2}+\tau^{2}}, \quad \Omega=\sqrt{u^{2}\left(\kappa^{2}+\tau^{2}\right)} .
$$

Thus, the curvature and torsion can be used to find a spiral as a local approximation to any space curve.

In addition to the intrinsic geometric parameters, we can also introduce $u \kappa$ (with $u \equiv|\mathbf{u}|$ ), the angular velocity on the tangential circle. It is different from the angular velocity $\Omega$ in the spiral because of the tilt of the tangential circle relative to the axis of the spiral: the equations for the spiral (14) show that $\Omega$ is the angular speed in the projection on the base plane, perpendicular to the axis. We will refer to $u \kappa$ as the curvature angular velocity.

For completeness, we note that a parametrization of the spiral locally approximating a trajectory passing through $\mathbf{r}\left(t_{0}\right)$ at time $t_{0}$ is given by

$$
\mathbf{r}(t)=\mathbf{r}_{0}+R\left[\mathbf{n}_{0} \times \mathbf{a} \sin \left(\Omega t^{\prime}\right)-\mathbf{n}_{0}\left(\cos \left(\Omega t^{\prime}\right)-1\right)\right]+\beta \Omega t \mathbf{a},
$$

where $t^{\prime}=t-t_{0}$. The unit vector $\hat{\mathbf{a}}=C_{a} \mathbf{n} \times(\mathrm{d} \mathbf{n} / \mathrm{d} s)$ (with $C_{a}$ a suitable normalization factor) points along the axis of the spiral.

Note that the vorticity of the velocity field that generates the curve points along the axis of the spiral, curl $\mathbf{u}=(0,0,2 \Omega)$.

In order to see the relation between the curvature and torsion and derivatives of the velocity field, we consider a stationary velocity field. A linear expansion of the velocity field around the point $\mathbf{x}$ then gives an overall translation with velocity $\mathbf{u}_{0}(\mathbf{x})$ and in the neighbourhood a variation $L \cdot \mathrm{d} \mathbf{x}$ with the shear matrix $L=\partial_{i} \mathbf{u}_{j}$. Decomposing the local shear into a symmetric $S=\frac{1}{2}\left(L+L^{T}\right)$ and antisymmetric part $A=\frac{1}{2}\left(L-L^{T}\right)$,

$$
S=\left(\begin{array}{ccc}
S_{1} & S_{2} & S_{3} \\
S_{2} & S_{4} & S_{5} \\
S_{3} & S_{5} & S_{6}
\end{array}\right), \quad A=\left(\begin{array}{ccc}
0 & A_{1} & A_{2} \\
-A_{1} & 0 & A_{3} \\
-A_{2} & -A_{3} & 0
\end{array}\right),
$$


and

$$
\mathbf{u}(\mathbf{x}+\mathrm{d} \mathbf{x})=\mathbf{u}_{0}(\mathbf{x})+(S+A) \mathrm{d} \mathbf{x}
$$

For a coordinate system with the main velocity along the $x$-direction, $\mathbf{u}_{0}(\mathbf{x})=\left|\mathbf{u}_{0}(\mathbf{x})\right| \mathbf{e}_{1}$, the curvature and torsion only depend on transverse shear components,

$$
\begin{aligned}
\kappa & =\sqrt{\frac{\left(S_{2}-A_{1}\right)^{2}+\left(S_{3}-A_{2}\right)^{2}}{\mathbf{u} \cdot \mathbf{u}}} \\
\tau & =\frac{-A_{3}}{|\mathbf{u}|}+S_{5}\left(2 \frac{\left(S_{2}-A_{1}\right)^{2}}{\kappa^{2}}-1\right)+\frac{S_{2}-A_{1}}{\kappa} \sqrt{1-\frac{\left(S_{2}-A_{1}\right)^{2}}{\kappa^{2}}}\left(S_{6}-S_{4}\right) .
\end{aligned}
$$

Note that while (20) contains only combinations of $S$ and $A$ as they arise in single offdiagonal components of the shear matrix, (21) also contains the symmetric combinations (in the second term). For a time-dependent flow, additional components need to be considered; substituting the time change of the velocity field for the Lagrangian acceleration, we obtain

$$
\kappa(t)=\frac{\left|\mathbf{u} \times \partial_{t} \mathbf{u}+\mathbf{u} \times[\mathbf{u} \cdot \nabla \mathbf{u}]\right|}{|\mathbf{u}|^{3}} .
$$

The significance of the partial time derivative will be shown below.

\section{Steady ABC flow}

As a step towards the turbulent situation, we consider trajectories in a steady flow with nontrivial streamlines. The flow is modelled after the classical ABC flow [17], except that bounding surface in the $x$-direction is introduced in order to model shear flows. The velocity field is

$$
\begin{aligned}
\mathbf{u}= & -\frac{A}{\pi} \operatorname{curl} \mathbf{e}_{z}(\sin \pi x \cos \pi y)+\frac{B}{\pi} \operatorname{curl} \mathbf{e}_{y}(\sin \pi x \cos \pi z) \\
& +\frac{C}{\pi} \operatorname{curl} \mathbf{e}_{x}(\cos \pi y+\sin \pi z) \\
= & \left(\begin{array}{c}
(A \sin \pi y+B \sin \pi z) \sin \pi x \\
A \cos \pi y \cos \pi x+C \cos \pi z \\
B \cos \pi z \cos \pi x+C \sin \pi y
\end{array}\right)
\end{aligned}
$$

with constant amplitudes $A, B$ and $C$. Incompressibility is ascertained by the representation as a curl of a vector field. A distribution of trajectory segments is shown in figure 2 .

Histograms of the curvature and torsion for these trajectory segments are shown in figure 3 The ones for the curvature show a maximum near $\kappa \sim 1$, a rapid drop off for larger values, and a slower one towards zero. The distribution for the torsion is fairly flat for small values and drops off rapidly for values in excess of about 1.

The representation of the curvature (20) actually suggests that the distribution has a linear increase for small values. For if $\xi_{2}$ and $\xi_{3}$ denote the combinations $S_{2}-A_{2}$ and $S_{3}-A_{3}$, normalised by the velocities, respectively, then the distribution for the curvature can be obtained from their distributions via

$$
P(\kappa)=\int \mathrm{d} \xi_{2} \int \mathrm{d} \xi_{3} \delta\left(\kappa-\sqrt{\xi_{2}^{2}+\xi_{3}^{2}}\right) P\left(\xi_{2}, \xi_{3}\right) .
$$




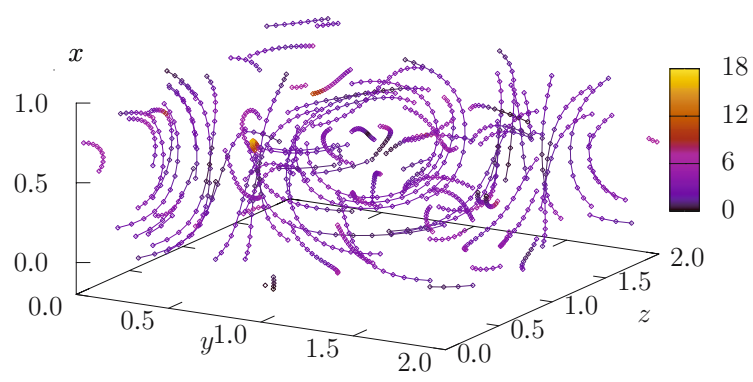

Figure 2. Trajectory segments of length 0.8 in dimensionless time in the $\mathrm{ABC}$ flow with parameters $A=B=1$, $C=0.8$. The colour coding shows the different curvatures along the trajectories. Some of the trajectories are fairly stretched out; a few are tightly wound spirals.

Introducing polar coordinates, $\xi_{2}=\xi \cos \phi$ and $\xi_{3}=\xi \sin \phi$, the representation becomes

$$
P(\kappa)=\kappa \int \mathrm{d} \phi P(\kappa \cos \phi, \kappa \sin \phi) .
$$

Hence, unless $P\left(\xi_{2}, \xi_{3}\right)$ vanishes for arguments approaching zero, the linear factor of $\kappa$ will dominate the small $\kappa$ behaviour.

\section{Turbulent flows}

In the laminar $\mathrm{ABC}$ flow, the length scales are determined by the vortices. In a turbulent flow they are determined by the scales of turbulent flows, down to the Kolmogorov scales [18]. Since the curvature depends on (a subset of) the gradients of the velocity field, and since the gradients also enter the dissipation, one may expect some correlation between the dissipation and curvature of the Lagrangian paths. In particular, we want to study a relation between curvature and the local one point dissipation, $\epsilon(\mathbf{x}, t)=\frac{v}{2}\left(\partial_{i} u_{j}+\partial_{j} u_{i}\right)^{2}$, of the form

$$
\langle\epsilon\rangle \sim \nu\langle\kappa|\mathbf{u}|\rangle^{2} \text {. }
$$

We study this relation for data from a direct numerical simulation (DNS).

The relevant statistics was collected in DNS of the Navier-Stokes equations on a fully periodic, cubic domain, at a resolution of $128^{3}$ points. A standard, fully dealiased pseudo-spectral
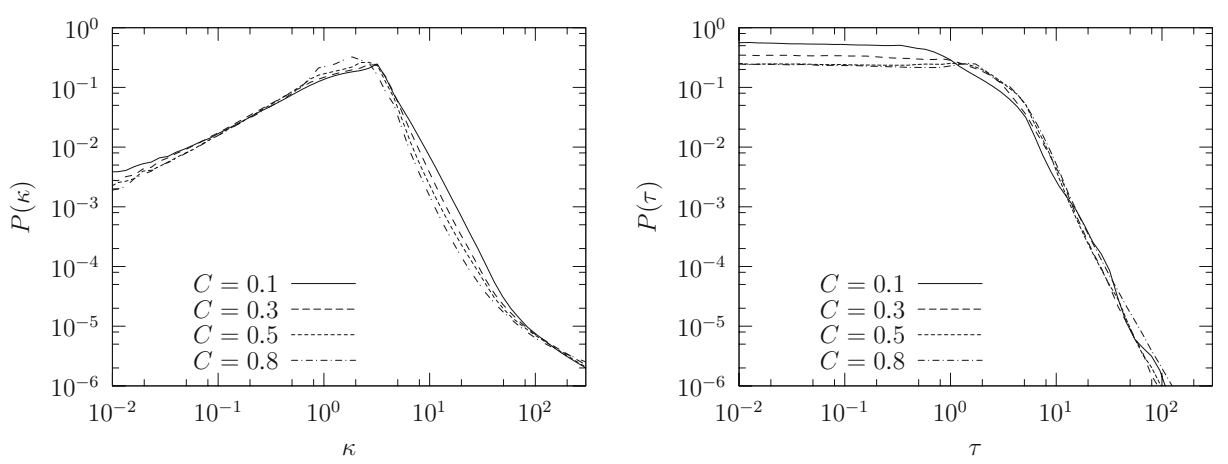

Figure 3. Curvature (left) and torsion (right) of trajectories in the ABC flow. For the statistics, short trajectory segments starting from points of a grid of mesh size 0.02 were computed. The parameters are $A=B=1$. 
Table 1. Parameters and properties of the numerical simulation.

\begin{tabular}{lc}
\hline Average energy & $\langle E\rangle=2.51$ \\
Average dissipation & $\epsilon=0.98$ \\
Size of the box & $L=2 \pi$ \\
Resolution & $128^{3}$ \\
Large eddy t/o time & $T \sim 5$ \\
Length of the simulation & $\sim 18 T$ \\
Number of Lagrangian trajectories & 10000 \\
$R E_{\lambda}$ & 95 \\
\hline
\end{tabular}

code was used [16, 19], in which a second-order Runge-Kutta scheme is implemented for time advancement. The large-scale energy input was provided by fixing the energy content of the first two Fourier shells[20]. Particle velocities for the Lagrangian integration were obtained by interpolation of the on-grid values by means of a third-order polynomial algorithm.

From the Navier-Stokes equation, the total time derivative (1) is determined by the pressure, the viscosity term and volume forces driving the flow,

$$
\mathrm{d} \mathbf{u} / \mathrm{d} t=-\nabla p / \rho+v \Delta \mathbf{u}+\mathbf{f} .
$$

Particle accelerations were determined by interpolation of the above quantities, while second derivatives were computed by centred finite differences of acceleration along the trajectories. As expected for developed turbulence [7, 10, 21], the main contribution to the total time derivative was found to be due to the pressure term. Simulations were carried out at different Reynolds numbers. To begin, we focus on a run at $R e_{\lambda}=95$ (see table 1).

For dimensional reasons, the quantity most directly related to energy dissipation is $u \kappa$, with $u \equiv|\mathbf{u}|$. The distributions of $\kappa$ and $u \kappa$ are shown in figure 4 . The curvature probability density function (pdf) exhibits a power-law behaviour for both large and small arguments. For small arguments, it is close to linear. The reasoning advanced in the last section around (25) is not directly applicable because of the contributions from the explicit time derivative. However, this difference only seems to be relevant for larger values of the curvature: if the explicit time dependence is omitted, and only the variations from the advective part are kept, as reflected in the definition of an advective curvature $u \kappa_{\nabla}=|\mathbf{u} \times \mathbf{u} \cdot \nabla \mathbf{u}| /|\mathbf{u}|^{\mathbf{2}}$, one finds that the probability distribution of this quantity is similar for small curvatures only (figure 4 , right).
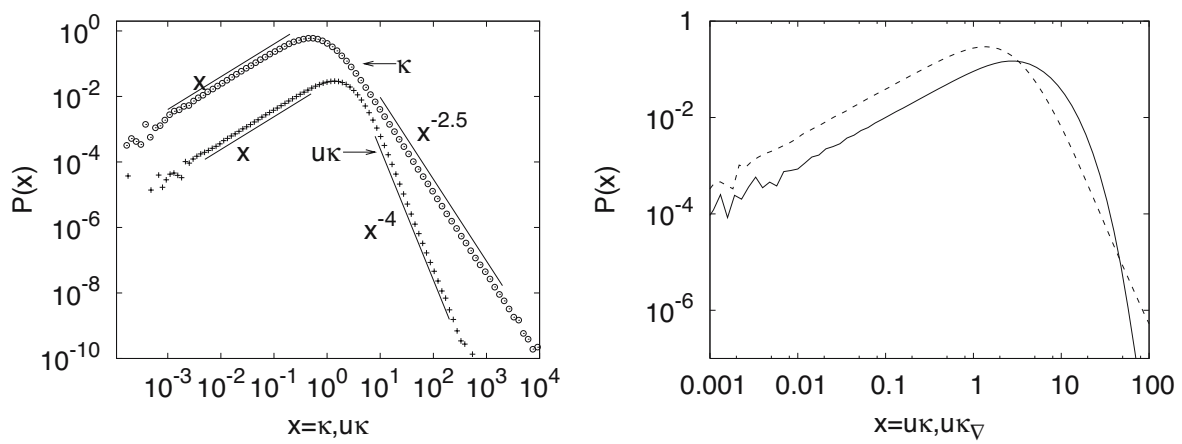

Figure 4. Left: Pdfs of curvature (circles) and curvature angular velocity $u \kappa$ (crosses). The distribution for $u \kappa$ was scaled down by 0.1 for better visibility. The distributions show a linear increase for small values, but different powers for large values. Right: to highlight the contributions of the time derivative in (22) to the curvature, we compare the pdfs for the curvature angular velocity $u \kappa$ (dashed line) and the advective curvature $u \kappa_{\nabla}$ (full line). The linear increase for small arguments is present in both, but their decay for larger values differs. 
Table 2. Correlation matrices, correlation coefficient and mean values for pairs formed from the curvature angular velocity $u \kappa$, the energy dissipation $\epsilon$ and the vorticity $\omega$. With $\langle\cdot\rangle$ denoting the ensemble average, the definitions of the measures are: centred second moment $s(a, b)=\langle(a-\langle a\rangle)(b-\langle b\rangle)\rangle$ and correlation coefficient $r(a, b)=s(a, b) / \sqrt{s(a, a) s(b, b)}$.

\begin{tabular}{lllllllll}
\hline$R e_{\lambda}$ & $a$ & $b$ & $s(a, a)$ & $s(b, b)$ & $s(a, b)$ & $r(a, b)$ & $\langle a\rangle$ & $\langle b\rangle$ \\
\hline 61 & $u \kappa$ & $\epsilon$ & 2.16 & 0.31 & 0.21 & 0.26 & 1.58 & 0.53 \\
& $\omega$ & $\epsilon$ & 13.5 & 0.31 & 0.92 & 0.45 & 5.51 & 0.53 \\
& $\omega$ & $u \kappa$ & 13.5 & 2.16 & 1.93 & 0.36 & 5.51 & 1.58 \\
71 & $u \kappa$ & $\epsilon$ & 3.14 & 0.37 & 0.29 & 0.27 & 1.84 & 0.56 \\
& $\omega$ & $\epsilon$ & 19.9 & 0.37 & 1.27 & 0.47 & 6.54 & 0.56 \\
& $\omega 5$ & $u \kappa$ & 19.9 & 3.14 & 2.82 & 0.36 & 6.54 & 1.84 \\
& $\omega \kappa$ & $\epsilon$ & 7.4 & 1.09 & 0.83 & 0.29 & 2.91 & 0.98 \\
& $\omega$ & $\epsilon$ & 51.4 & 1.09 & 3.35 & 0.43 & 10.5 & 0.98 \\
& $\omega$ & ик & 51.4 & 7.37 & 7.18 & 0.37 & 10.5 & 2.89 \\
\hline
\end{tabular}

For larger arguments, the pdf for the curvature $\kappa$ decays with an exponent of -2.5 , while that for the curvature angular velocity $u \kappa$ decays more quickly, with an exponent of -4 . Here, the contribution from the time derivative is significant, for the decay of the advective derivative is more compatible with a stretched exponential, rather than an algebraic decay.

The relation between the curvature and dissipation is studied further in table 2 and figure 5 . Figure 5 shows the joint pdf for curvature and dissipation as well as the joint pdf between the curvature angular velocity and the dissipation. There is a clear maximum, which may be used to relate mean curvature and mean dissipation. The logarithmic representation emphasises the tails in the distribution, and the linear one shows that the fluctuations around the maximum are large. The contour lines around the maximum are fairly round and not elongated much along the diagonal, reminiscent of the pdf that could be obtained from a product of two independent
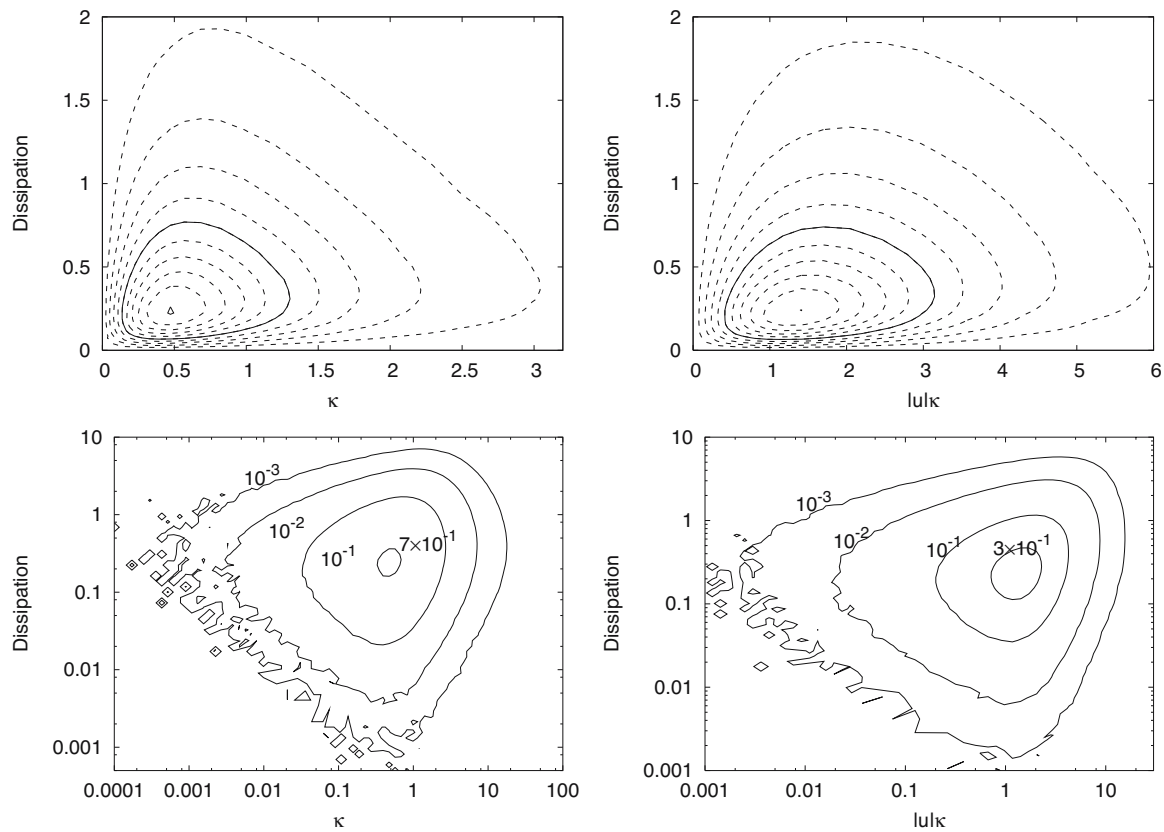

Figure 5. Contour plot of the joint pdf of curvature and dissipation (left) and modified curvature rate and dissipation (right). In the top row the figures are shown in a linear scaling, and in the bottom row they are in logarithmic scaling. 

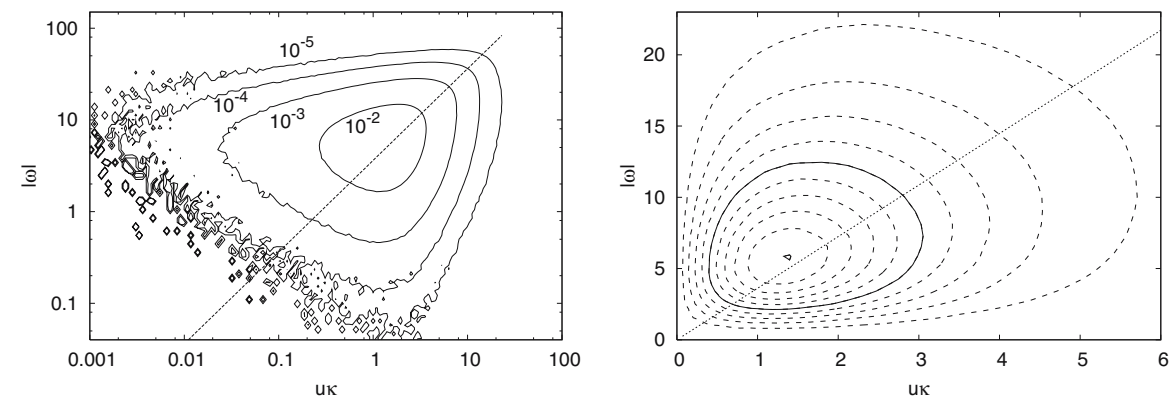

Figure 6. Contour plots of the joint pdf of the absolute value of the vorticity and the curvature angular velocity, once on a logarithmic scale (left) and a linear scale (right). The dashed straight line passes through the origin and the maximum and indicates the linear correlation the correlation coefficient is probing for.

pdfs with a single maximum. Indeed, the correlation coefficient in table 2 does not exceed values of 0.3 , indicating a weak correlation only. This implies that, for trajectories collected at fixed $R_{\lambda}$, it is not possible to conclude from the observation of a tightly wound spiral (large $\kappa)$ that it is passing through a region of high dissipation.

As an aside, we note that the joint distribution between the dissipation and the square of the curvature angular velocity $u \kappa$ does not show a pointed maximum as in figure 5 . The reason lies in the behaviour of probability distributions under transformations: going from $x$ to $y=x^{2}$, the distribution becomes $P(y)=p(\sqrt{y}) /(2 \sqrt{y})$. Together with the linear behaviour for small curvatures, the distribution in $(u \kappa)^{2}$ becomes flat for small curvatures.

To study the relation of the local path geometry to vorticity, we analyse the correlations between the absolute value of the vorticity $\omega=|\boldsymbol{\omega}|=\mid$ curl $\mathbf{u} \mid$ and the curvature angular velocity $u \kappa$, see figure 6 . The joint pdf is fairly wide, but there is a clear maximum and the correlation coefficient is acceptable with 0.37 . The logarithmic presentation highlights the wide tails and the small-scale contributions. Other measures of vorticity and the curvature of the trajectory do not seem to provide stronger correlations. For instance, if the angular velocity of the spiral is determined from curvature and torsion as in (2), then slowly decaying algebraic tails in the torsion prevent the determination of the second moment and hence the correlation coefficient. Projecting the vorticity on the axis of the spiral introduces an ambiguity in the sign, which emphasises the region of small vorticity. A priori, neither measure is singled out compared to others, since the details of the particle paths do not only depend on the velocity field, but also on the initial conditions with which the particles enter the region of the vortex.

All the preceding diagrams were for a run at a single Reynolds number, $R e_{\lambda}=95$. Varying the Reynolds number results in a change in small-scale statistics, since the dissipative range is more extended for lower $R e_{\lambda}$. A change is therefore expected also in the statistics of curvature. To investigate this point, we performed further simulations with varying kinematic viscosity. Figure 7 shows the pdfs for runs at different $R e_{\lambda}$. The inset shows a linear relation between the maxima in the distributions of curvature angular velocity $u \kappa$ and vorticity $\omega$, obtained for different Reynolds numbers $R_{\lambda}$. The maxima are related by

$$
\max p(\omega) \approx 4 \max p(u \kappa) .
$$

This confirms the expectation that the curvature, in the particular combination of velocity times curvature, can be used to extract information about gradients, in this case the most likely value of the angular momentum. Thus, while there are only weak correlations between $u \kappa$ and $\omega$ at fixed $R_{\lambda}$, there is a clear scaling of the maxima in the joint pdf with $R_{\lambda}$. 

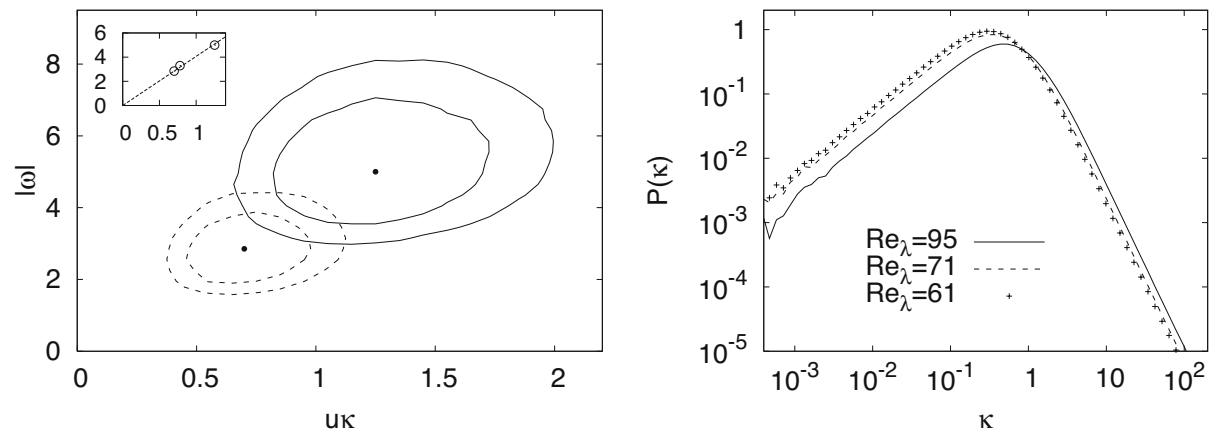

Figure 7. Left: contour plots of the $90 \%$ and $80 \%$ levels of the joint pdf of curvature angular velocity and angular velocity for Reynolds numbers $R e_{\lambda}=61$ and $R e_{\lambda}=95$ respectively. The inset also includes data for $R e_{\lambda}=71$ and suggests the linear relation (28) between the maxima. Right: Pdfs of curvature at different $R e_{\lambda}$. A shift of the maximum towards larger values can be observed, as for $u \kappa$, at increasing $R e_{\lambda}$, while the shape of the distribution is preserved.

\section{Final remarks}

The study has shown that in particular the curvature of the particle paths can be used to extract statistical information about velocity gradients from a single trajectory. The relation between the curvature of a trajectory and the presence of a strong vorticity is one of maximal likelihood (the joint pdf has a maximum there), and there is a systematic variation in the position of the maximum with the Reynolds number, leading to a linear relation between the curvature angular momentum $u \kappa$ and the local angular momentum $\omega$. However, at fixed Reynolds numbers the fluctuations around the maximum are large, and the correlations between local and instantaneous values of $u \kappa$ and $\omega$ are weak.

\section{Acknowledgement}

This work was supported by the Deutsche Forschungsgemeinschaft.

\section{References}

[1] Ott, S. and Mann, J., 2000, An experimental investigation of the relative diffusion of particle pairs in threedimensional turbulent flow. Journal of Fluid Mechanics, 422, 207-223.

[2] La Porta, A., Voth, G.A., Crawford, A.M., Alexander, J. and Bodenschatz, E., 2001, Fluid particle accelerations in fully developed turbulence. Nature, 409, 1017.

[3] Voth, G.A., La Porta, A., Crawford, A.M., Bodenschatz E. and Alexander, J., 2001, A silicon strip detector system for high resolution particle tracking in turbulence. Review of Scientific Instruments, 72, 4348.

[4] Mordant, N. and Pinton, J.F., 2001, Measurement of Lagrangian velocity in fully developed turbulence. Physical Review Letters, 87, 214501.

[5] Mordant, N., Metz, P., Pinton, J.F. and Michel, O., 2005, Acoustical technique for Lagrangian velocity measurement. Review of Scientific Instruments, 76, 025105.

[6] Mordant, N., Crawford, A.M. and Bodenschatz, E., 2004, Three-dimensional structure of the Lagrangian acceleration in turbulent flows. Physical Review Letters, 93, 214501.

[7] Voth, G.A., La Porta, A., Crawford, A.M., Alexander, J. and Bodenschatz, E., 2002, Measurement of particle accelerations in fully developed turbulence. Journal of Fluid Mechanics, 469, 121-160.

[8] Reynolds, A.M., Mordant, N., Crawford, A.M. and Bodenschatz, E., 2005, On the distribution of Lagrangian accelerations in turbulent flows. New Journal of Physics, 7, 58.

[9] Mordant, N. , Leveque, E. and Pinton, J.F., 2004, Experimental and numerical study of the Lagrangian dynamics of high Reynolds turbulence. New Journal of Physics, 6, 116.

[10] Biferale, L., Boffetta, G., Celani, A., Devenish, B.J., Lanotte, A. and Toschi, F., 2004, Multifractal statistics of Lagrangian velocity and acceleration in turbulence. Physical Review Letters, 93, 064502. 
[11] Friedrich, R., 2003, Statistics of Lagrangian velocities in turbulent flows. Physical Review Letters, $90,084501$.

[12] Shaw, R.A., 2003, Particle-turbulence interactions in atmospheric clouds. Annual Review of Fluid Mechanics, 35, 183-227.

[13] Pumir, A., Shraiman, B.I. and Chertkov, M., 2000, Geometry of Lagrangian dispersion in turbulence. Physical Review Letters, 85, 5324-5327.

[14] Falkovich, G., Gawedzki, K. and Vergassola, M., 2001, Particles and fields in fluid turbulence. Reviews of Modern Physics, 73, 913-975.

[15] Lüthi, B., Tsinober, A. and Kinzelbach, W., 2005, Lagrangian measurement of vorticity dynamics in turbulent flow. Journal of Fluid Mechanics, 528, 87-118.

[16] Biferale, L., Boffetta, G., Celani, A., Lanotte, A. and Toschi, F., 2005, Particle trapping in three-dimensional fully developed turbulence. Physics of Fluids, 17, 021701.

[17] Dombre, T., Frisch, U., Greene, J., Henon, M., Mehr, A. and Soward, A., 1986, Chaotic streamlines in the ABC flows. Journal of Fluid Mechanics, 167, 353-391.

[18] Frisch, U. Turbulence, University Press, Cambridge, 1995.

[19] Canuto, C., Hussaini, M.Y., Quarteroni, A. and Zang, T.A., Spectral Methods in Fluid Dynamics, New York: Springer-Verlag, 1988.

[20] Chen, S., Doolen, G.D., Kraichnan, R.H. and She, Z.-S., 1993, On statistical correlations between velocity increments and locally averaged dissipation in homogeneous turbulence. Physics of Fluids, A 5, 458-463.

[21] Vedula, P. and Yeung, P.K, 1999, Similarity scaling of acceleration and pressure statistics in numerical simulations of isotropic turbulence, Physics of Fluids, 11, 1208-1220. 